Cahiers Charlevoix

Études franco-ontariennes
Cahiers Charlevoix Études franco-ontariennes

or Crevereerie of:

\title{
Le Nouveau Visage de l'ambition en Ontario français. Le cas du Nord-Est
}

\section{Simon Laflamme}

Volume 8, 2010

URI : https://id.erudit.org/iderudit/1039318ar

DOI : https://doi.org/10.7202/1039318ar

Aller au sommaire du numéro

\section{Éditeur(s)}

Société Charlevoix

Presses de l’Université d’Ottawa

\section{ISSN}

1203-4371 (imprimé)

2371-6878 (numérique)

Découvrir la revue

Citer cet article

Laflamme, S. (2010). Le Nouveau Visage de l'ambition en Ontario français. Le cas du Nord-Est. Cahiers Charlevoix, 8, 77-110.

https://doi.org/10.7202/1039318ar
Résumé de l'article

Simon Laflamme présente le nouveau visage de l'ambition de la jeunesse franco-ontarienne. Les quatre grandes questions examinées dans son étude confirment une ressemblance de plus en plus marquée entre les francophones et les anglophones, tant les projets d'étude ou de carrière des uns s'apparentent, à quelques différences près, à ceux des autres. Selon l'auteur, cette transformation, opérée au cours des quinze dernières années, est à mettre en lien avec des tendances des sociétés industrielles avancées, notamment l'homogénéisation, attribuable à la postindustrialisation, et les moyens de communication de masse qui en sont le corollaire. Or, " [l]'homogénéisation des populations, sans que chacune d'elles dispose de ses institutions propres, est simple négation des particularismes, et assimilation aux messages et aux conditions de vie de l'ensemble qui est le mieux pourvu au plan institutionnel », remarque-t-il. Dans ce contexte, une société qui sait se doter d'un « système institutionnel nécessaire aux sociétés postindustrielles, et donc qui soit commun à chacune d'elles, mais qui simultanément assure leur différenciation » rend moins probable l'assimilation. Voilà ce qui affermirait la nouvelle position des aspirations de la jeunesse franco-ontarienne et qui maintiendrait toujours les jeunes des Premières Nations en marge, eux qui « peinent à se projeter vers l'université ou le font à certains égards s'ils admettent la nécessité de l'anglicité du monde ».
Ce document est protégé par la loi sur le droit d'auteur. L’utilisation des services d'Érudit (y compris la reproduction) est assujettie à sa politique d'utilisation que vous pouvez consulter en ligne.

https://apropos.erudit.org/fr/usagers/politique-dutilisation/ 


\title{
Le Nouveau Visage de l'ambition en Ontario français. Le cas du Nord-Est
}

\author{
SimON LAFLAMME \\ Département de sociologie \\ Université Laurentienne
}




\section{SOMMAIRE}

I - L'ambition et les Franco-Ontariens 79

II - Méthodologie et Profil de L’ÉCHANTILlon 90

III - Des ASPIRATIONS COMPARABLES 92

A. Les aspirations éducationnelles 92

B. Les aspirations professionnelles 94

C. Parenthèses sur les aspirations professionnelles 98

IV - ASPIRATIONS ET REPRÉSENTATIONS LINGUISTIQUES 99

V - D'AUTRES DÉTERMINANTS

DES ASPIRATIONS PROFESSIONNELLES 103

VI - INTERPRÉTATION ET CONCLUSION 106 


\section{Le Nouveau Visage de l'ambition en Ontario français. Le cas du Nord-Est ${ }^{1}$}

\section{I - L'ambition et les Franco-Ontariens}

Les aspirations éducationnelles et professionnelles des jeunes ont beaucoup attiré l'attention à compter du début des années 1970 . La sociologie en était venue à comprendre que, à travers ces aspirations, se dessinent le destin de chacun des jeunes et, par conséquent, celui de toute la société à l'intérieur de laquelle les jeunes évoluent. Une perspective libérale voulait que l'on devienne ce que l'on a voulu être. Une position marxisante ne contestait pas cette corrélation entre la manière dont on conçoit son avenir et cet avenir lui-même, mais elle soutenait avec véhémence que l'ambition variait selon la classe sociale, que les enfants des familles favorisées s'orientaient davantage que les autres vers les études universitaires et vers les professions qui accordent le plus de privilèges. Étudier les aspirations, donc, c'était entrevoir le futur de chacun et celui de toute une population, c'était mettre en évidence une des principales sources de discrimination sociale.

C'est dans cet esprit que, au Canada, Raymond Breton a rédigé Social and Academic Factors in the Career Decisions of Canadian $Y_{\text {Youth }}{ }^{2}$ et Le Rôle de l'école et de la société dans le choix d'une carrière chez la jeunesse canadienne ${ }^{3}$, que Marion R. Porter, John

1. Cette recherche a été rendue possible grâce au financement assuré par la Commission de formation du Nord-Est (CFNE).

2. Social and Academic Factors in the Career Decisions of Canadian Youth, Department of Manpower and Immigration of Canada, Program Development Service, 1972.

3. Le Rôle de l'école et de la société dans le choix d'une carrière chez la jeunesse canadienne. Une étude auprès des étudiants du secondaire, Ottawa, Ministère de la Main-d'œuvre et de l'Immigration du Canada, Établissement des programmes, 1972. 
Porter et Bernard R. Blishen ont écrit Does Money Matter? ${ }^{4}$, tous des ouvrages abondamment cités. C'est encore dans cet esprit qu'a été créé le groupe Aspirations scolaires et orientations professionnelles des élèves (AsOPE) qui publiera Les Cahiers d'ASOPE. Ce groupe a mené une vaste enquête en 1971 et 1972. En 1979, Louise Laforce et ses collaborateurs ${ }^{5}$, en s'appuyant sur les données de cette enquête, observaient que, dans des populations d'élèves, les Franco-Ontariens étaient plus nombreux que les francophones et les anglophones du Québec aussi bien que les anglophones de l'Ontario à priser les professions les plus en vue. En comparant les élèves de secondaire $v$ au Québec et ceux de douzième année, en Ontario, qui sont à un niveau équivalent, les données révélaient, en effet, que $42 \%$ des francophones de l'Ontario aspiraient aux professions qui appartenaient à la catégorie « élevées ${ }^{6} »$; cette proportion était de $31 \%$ pour les Anglo-Ontariens, de $33 \%$ et de $21 \%$ respectivement pour les anglophones et les francophones du Québec. Les visées de ces jeunes étaient plutôt optimistes à l'égard des études quoique un peu moins chez les Franco-Québécois que chez les autres : au Québec, $65 \%$ des francophones et $77 \%$ des anglophones avaient l'intention de faire des études postsecondaires ; en Ontario, la

4. Does Money Matter ? Prospects for Higher Education, Toronto, Institute for Behavioural Research, York University, 1973. L'ouvrage sera réédité en 1979 avec un sous-titre plus circonscrit : Does Money Matter? Prospects for Higher Education in Ontario (Toronto, Macmillan et Institute of Canadian Studies of Carleton University, The Carleton Library, $\left.\mathrm{n}^{\circ} 110\right)$.

5. Louise Laforce, en collaboration avec Pierre W. Bélanger, Pierre Roberge et Guy Rocher, Les Aspirations scolaires au Québec et en Ontario : des observations des enquêtes ASOPE et SOSA, Les Cahiers d'ASOPE (Aspirations scolaires et orientations professionnelles des étudiants), vol. vI, Québec et Montréal, Faculté des sciences de l'éducation de l'Université Laval et Département de sociologie de l'Université de Montréal, 1979.

6. Les professions ont été regroupées en trois catégories : élevées, moyennes et faibles. " [L]a strate supérieure compre[nd] surtout les propriétaires et gérants d'entreprise, les membres des professions libérales ; la strate moyenne group[e] quelques propriétaires et gérants d'entreprise de moindre importance, les secrétaires et sténographes, vendeurs (immeubles, courtiers en valeur, publicité), certains contremaîtres dans l'industrie, les ouvriers spécialisés, etc. ; la strate inférieure [a] pour principaux représentants [...] les ouvriers semi-spécialisés, les manœuvres, les agriculteurs, les pêcheurs, les bûcherons, etc. » (ibid., p. 17). 
statistique était de $75 \%$ pour les francophones et de $72 \%$ pour les anglophones.

Ces résultats étaient intéressants à maints égards. Premièrement, la théorie pouvait expliquer assez aisément que les francophones du Québec, au début des années 1970, fussent proportionnellement moins nombreux que les anglophones de la même province à se tourner vers les emplois les plus valorisés puisqu'ils étaient défavorisés sur le plan socioéconomique, mais elle pouvait moins bien rendre compte de la récurrence des hautes ambitions des Franco-Ontariens puisque leur situation n'était pas avantagée en comparaison à celle des Anglo-Ontariens. Deuxièmement, les aspirations témoignaient dans l'ensemble d'une faible corrélation avec l'origine familiale; elles correspondaient souvent à des métiers plus élevés que ceux qu'exerçaient les parents, et pour les francophones de l'Ontario davantage que pour les autres, pour ceux du Québec, moins que pour les autres ; la même logique prévalait pour l'instruction : on envisageait communément des études postsecondaires même si les parents n'en avaient pas fait. Troisièmement, la structure des professions du groupe d'appartenance était peu associée à l'ensemble des aspirations, et cela, très nettement chez les Franco-Ontariens : la structure des emplois souhaités ne ressemblait pas à celle qui se donnait à l'observation au moment où furent déclarées les aspirations. Ainsi, non seulement la classe sociale de la famille d'origine ou la position sociale du groupe auquel on appartenait étaient peu déterminantes de la profession que voulait exercer le jeune, mais il semblait aussi que cette profession fût définie en tenant peu compte de la société qui accueillerait les travailleurs de demain. Quatrièmement, on pouvait deviner que les plans relativement à l'emploi et à l'instruction ne se traduisaient pas forcément de façon concrète ; ce qu'allait attester plus tard de façon éloquente un rapport de Stacy Churchill, Saeed Quazi et Normand Frenette ${ }^{7}$ dans lequel

7. Stacy Churchill, Saeed Quazi et Normand Frenette, Éducation et besoins des Franco-Ontariens. Le Diagnostic d'un système d'éducation, Toronto, Conseil de l'éducation franco-ontarienne, 1985, vol. 1 et 2. 
il serait démontré que les jeunes de l'Ontario français ont $50 \%$ moins de chances que les autres de faire des études postsecondaires.

Cette recherche, par conséquent, invitait à une certaine suspicion à l'endroit de la théorie de la détermination et suscitait la curiosité pour le cas franco-ontarien.

La suspicion sera toutefois timorée tant la foi en la théorie est grande. Des dizaines, des centaines de travaux dans le monde seront menés dans une logique de détermination de la classe sociale. Pierre Bourdieu ${ }^{8}$ fera école avec ses concepts de capital culturel, de capital symbolique, de capital linguistique et avec sa notion d'habitus. Le principe est simple: plus il y a de culture dans la famille de laquelle on provient, mieux on s'y exprime et plus son statut est prestigieux, alors meilleures sont les chances de bien réussir à l'école et dans tout le système d'éducation ; ces capitaux génèrent un habitus, un mode de comportement, des attitudes qui favorisent les enfants des classes supérieures. Combien de fois ne verra-t-on pas d'allusion à cette position ! Il faudra d'innombrables travaux où la théorie ne trouve qu'une confirmation partielle pour qu'on en vienne à réellement relativiser cette trop belle vision du monde. Pendant longtemps, l'interprétation des données insistera sur la partie qui donne crédit à la théorie en occultant, si imposant cela soit-il, tout ce qui l'invalide ou tout ce qui invite à la nuance. Dans des travaux récents, on aperçoit cette relativisation. Une étude de Nancy E. Hill et de ses collaborateurs ${ }^{9}$, par exemple, montre que le lien est ténu entre les aspirations des enfants et l'instruction, la profession

8. Pierre Bourdieu et Jean-Claude Passeron, Les Héritiers : les étudiants et la culture, Paris, Minuit, 1964 ; Pierre Bourdieu et Jean-Claude Passeron, La Reproduction. Éléments pour une théorie du système d'enseignement, Paris, Minuit, 1970 ; Pierre Bourdieu, La Distinction. Critique sociale du jugement, Paris, Minuit, 1979 ; Pierre Bourdieu, Homo Academicus, Paris, Minuit, 1987 ; Pierre Bourdieu, La Noblesse d'État. Grandes écoles et esprit de corps, Paris, Minuit, 1989.

9. Nancy E. Hill, Domini R. Castellino, Jennifer E. Lansford, Patrick Nowlin, Kenneth A. Dodge, John E. Bates et Gregory S. Pettit, «Parent Academic Involvement as Related to School Behavior, Achievement, and Aspirations : Demographic Variations Across Adolescence », Child Development, vol. 75, n 5, septembre 2004, p. 14911509. 
ou le revenu des parents, les corrélations ( $\mathrm{r}$ ) étant respectivement de 0,31,0,32 et 0,36. Une analyse de Barbara Signer et Deborah Saldana ${ }^{10}$ note, entre la scolarité qu'envisage l'enfant et le niveau d'instruction des parents, des $\chi^{2}$ qui ne sont pas attribuables au hasard, mais qui font nettement place à d'autres sources de détermination : les valeurs sont de 10,98 dans le tableau qui se rapporte à la mère et de 5,26 dans celui qui concerne le père. Une recherche de Wei-Cheng Mau et Lynette Heim Bikos ${ }^{11}$ trouve, dans une analyse de régression, un faible coefficient standardisé (ß) de 0,64 entre la combinaison de la scolarité et de la profession des parents, d'une part, et les projets éducationnels de leur enfant, d'autre part. D'autres variables indépendantes seront sollicitées pour rendre compte de l'aspiration des jeunes : l'ethnie, la race, le sexe, la région, l'école elle-même, comme pour combler les vides que produisent les analyses en fonction de l'origine de classe sociale tout en maintenant la foi dans une logique de discrimination et en des modélisations causalistes. Elles montreront toutes que ces variables indépendantes ont une incidence, mais faible.

Pour ce qui est de la curiosité que provoquait le cas francoontarien, les analyses se sont surtout penchées sur les caractéristiques des élèves ${ }^{12}$. Elles ont essentiellement tenté de découvrir les facteurs qui étaient susceptibles de faire varier les aspirations. On le voit, la logique déterministe persistait, inspirée qu'elle était par une quête des causes de la différenciation. Mais on parvenait mal à rendre compte du phénomène. Laforce et ses collaborateurs ${ }^{13} \mathrm{~s}$ 'étaient étonnés que les francophones de l'Ontario et ceux du Québec fussent fils et filles d'ouvriers et de

10. Barbara Signer et Deborah Saldana, «Educational and Career Aspirations of High School Students and Race, Gender, Class Differences », Race, Gender and Class, vol. 8, $\mathrm{n}^{\circ} 1,2001$, p. 22-34.

11. Wei-Cheng Mau et Lynette Heim Bikos, «Educational and vocational aspirations of minority and female students : A longitudinal study », Journal of Counseling and Development, vol. 78, $\mathrm{n}^{\circ}$ 2, printemps 2000, p. 186-194.

12. Pierre Poirier, Évelyn Gagné et Maurice Lapointe, Le Profil de l'étudiant franco-ontarien quant à son intentionnalité de poursuivre des études post-secondaires, Ottawa, Faculté d'éducation, Université d'Ottawa, 1987.

13. Louise Laforce et al., Les Aspirations scolaires au Québec et en Ontario, op. cit. 
manœuvres et que les projets de carrière des premiers dépendissent moins de « la situation sociale héritée de leurs pères ${ }^{14} »$. Ils écrivaient :

Les $[\mathrm{F}]$ ranco- $[\mathrm{O}]$ ntariens chercheraient donc à dépasser la condition sociale et économique de leurs parents en se scolarisant davantage pour parvenir à des carrières d'administrateurs et de professionnels dans différents domaines. Leurs aspirations élevées peuvent ainsi s'interpréter comme un désir de mobilité sociale à l'intérieur d'un système de classes où traditionnellement leurs pères se situaient en général aux échelons inférieurs. Dans une société où plusieurs minorités ethniques et linguistiques co-existent à côté d'une majorité d'origine britannique, les obstacles à la mobilité sociale sont peut-être moins perçus comme une conséquence inéluctable de l'origine ethnique. Et, en Ontario, les francophones bénéficient d'un enseignement dans leur propre langue, privilège dont ne jouissent pas les étudiants d'origine allemande ou italienne par exemple, deux communautés qui sont d'importance à peu près égale à la communauté francophone dans cette province. Ce dernier facteur n'est probablement pas étranger aux attentes élevées des jeunes francophones face à leur avenir scolaire et professionnel ${ }^{15}$.

Ils interprétaient donc le caractère inusité de l'ambition des Ontariens d'expression française comme un « désir de mobilité » ascendante ; pour eux, cette ambition était possible parce que l'Ontario comptait plusieurs « minorités ethniques et linguistiques », qu'il n'était donc pas dichotomisé comme le Québec entre deux groupes, d'origine, l'un, française, l'autre, britannique; cette ambition était aussi favorisée par un système scolaire qui admettait l'enseignement en français. Mais cette interprétation s'avérait peu satisfaisante. D'abord, on ne peut pas vraiment expliquer les fortes aspirations éducationnelles et professionnelles par un désir de mobilité ; le raisonnement est ainsi, pour le moins, tautologique. Deuxièmement, on peut difficilement soutenir que le caractère pluriel du minoritaire accroît la probabilité qu'il développe de

14. Ibid., p. 157.

15. Ibid., p. 157-158. 
fortes aspirations ; il est des groupes minoritaires, dans des sociétés où les minorités sont nombreuses, auxquels l'avenir est loin d'apparaître prometteur ; et puis la situation québécoise était moins duelle qu' on ne le laisse entendre dans la citation : Italiens, Irlandais, Amérindiens, Polonais et autres Juifs ou Asiatiques habitaient la Belle Province et pluralisaient 1'ensemble minoritaire, ou, plus exactement, la population non dominante. Troisièmement, les francophones du Québec pouvaient, eux aussi, s'instruire dans leur langue. Il faut ajouter à ces commentaires que le « désir de mobilité » ascendante ne donne pas automatiquement lieu à l'exercice des professions convoitées. Toute aspiration élevée chez le jeune ne correspond pas à une juste évaluation des aptitudes personnelles et des possibilités qui s'offrent à soi. À cet égard, on ne pourra pas donner entièrement tort aux commentateurs qui qualifieront les aspirations juvéniles de réalistes quand il s'agira de celles des Franco-Québécois et d'irréalistes lorsqu'elles se rapporteront aux Franco-Ontariens - Normand Frenette et Saeed Quazi parleront d' « idéalisme peu pragmatique $^{16} \gg$. Il était moins utile, cependant, de savoir si ces aspirations étaient ou non réalistes que de comprendre le rôle qu'elles jouaient dans les consciences populaires. Car si illusoires qu'elles fussent, elles ne pouvaient pas ne pas participer d'une vision du monde. Les déclarer réalistes ou irréalistes, ce n'était pas en donner la signification ; c'était porter sur elles un jugement. Certes on pouvait dire des projets professionnels des francophones du Québec qu'ils étaient réalistes si l'on entendait par là qu'ils correspondaient mieux à la situation socioéconomique de leurs

16. Frenette et Quazi mettent en parallèle les études sur les aspirations et celles qui examinent les besoins et les préférences des Franco-Ontariens dans lesquelles on note un intérêt marqué pour les programmes bilingues et ils écrivent : " Ces études [...] n'arrivent pas à expliquer pourquoi les répondants et les répondantes témoignent d'un idéalisme peu pragmatique en matière d'aspirations scolaires et professionnelles et d'un pragmatisme peu idéaliste en matière de choix des établissements scolaires » («L'Accessibilité aux études postsecondaires pour les francophones de l'Ontario », dans Gratien Allaire et Anne Gilbert (dir.), Francophonies plurielles. Communications choisies, Colloque du regroupement pour la recherche sur la francophonie canadienne organisé dans le cadre du congrès annuel de l'ACFAs (Chicoutimi, 1995, et Montréal, 1996), Sudbury, Institut franco-ontarien, « Fleur de trille», 1998, p. 257). 
parents, mais la question se posait tout de même de savoir ce qui empêchait ces jeunes d'envisager l'avenir autrement que ne l'avaient fait leurs parents. Pareillement, on pouvait dire que les intentions des jeunes franco-ontariens étaient irréalistes en ce qu'elles témoignaient d'une certaine utopie, mais la question se posait de savoir pourquoi cette utopie intervenait dans la conscience collective.

C'est à cette dernière question que nous avons tenté de répondre dans L'Ambition démesurée ${ }^{17}$, il y a plus de quinze ans, alors que nous étudiions la grande région du Nord-Est, grosso modo de Sault-Sainte-Marie et de Hearst au Témiscamingue. Nous avions alors observé que le fait d'envisager des études postsecondaires ou de se tourner vers les professions les plus élevées de l'échelle sociale ne constituait pas normalement un projet bien arrêté. En effet, à quelque niveau de scolarité où nous fîmes l'observation, le nombre de jeunes qui annonçaient qu'ils poursuivraient leurs études au-delà du secondaire était toujours d'au moins 88,6 \% ${ }^{18}$ et l'ensemble de ceux qui caressaient le rêve d'occuper les emplois de niveau supérieur, d'au moins $40 \%$, sauf en dixième année ${ }^{19}$. Or, ces ambitions communément élevées n'empêchaient pas qu'on abandonnât ses études, les nombres d'inscrits dans les écoles diminuant nettement de la huitième à la treizième année ; autrement dit, on peut avoir de hautes aspirations et ne pas demeurer à l'école, et être à l'école c'est, la plupart du temps, avoir de hautes aspirations. Nous avons mis cette information en relation avec des données sur la manière dont les

17. Simon Laflamme et Donald Dennie, avec la collaboration d'Yvon Gauthier, L'Ambition démesurée. Enquête sur les aspirations et les représentations des étudiants et des étudiantes francophones du Nord-Est de l'Ontario, Sudbury, Prise de Parole/ Institut franco-ontarien, 1990, $194 \mathrm{p}$.

18. $8^{\mathrm{e}}: 93,9 \% ; 9^{\mathrm{e}}: 95,6 \% ; 10^{\mathrm{e}}: 88,6 \% ; 11^{\mathrm{e}}: 93,4 \% ; 12^{\mathrm{e}}: 97,3 \% ; 13^{\mathrm{e}}$ : 98,3\%. Ibid., p. 90. Vers la fin des années 1970, Raymond Mougeon, Katherine Rehner et Nathalie Alexandre ont obtenu des proportions un peu plus faibles (Le Français parlé en situation minoritaire : choix de langue, identité linguistique et variation linguistique parmi les élèves des écoles de langue française dans cinq communautés franco-ontariennes. Rapport final préparé pour le ministère de l'Éducation de l'Ontario, 2005).

19. $8^{\mathrm{e}}: 42,7 \% ; 9^{\mathrm{e}}: 44,2 \% ; 10^{\mathrm{e}}: 29,3 \% ; 11^{\mathrm{e}}: 46,3 \% ; 12^{\mathrm{e}}: 43,8 \% ; 13^{\mathrm{e}}$ : $62,3 \%$. Ibid., chapitre IV. 
jeunes franco-ontariens percevaient la francité et l'anglicité et dont ils se positionnaient eux-mêmes dans cet univers linguistique. Les analyses ont permis de découvrir, notamment vers la dixième année de scolarité, une forte admiration pour ce qui est anglais et un certain mépris pour ce qui est français. Nous avons alors soulevé l'hypothèse que les fortes aspirations juvéniles des Franco-Ontariens, sur les plans éducationnel et professionnel, étaient une manière de conjurer un intense sentiment de minoritude. Vouloir occuper les emplois qui se situent au sommet de l'échelle sociale, c'était se concevoir comme objectivement noble, c'était se projeter dans les postes qui font l'admiration de tous, francophones comme anglophones. Mais la fin exorcisante de cette ambition était souvent plus grande que l'ambition elle-même, de sorte que bon nombre de jeunes ne se donnaient pas les moyens d'en matérialiser les termes. Dans le même esprit, cette ambition était souvent trop grande, trop peu associée à des aptitudes réelles, de sorte que le jeune qui l'entretenait était appelé à s'en détourner nettement plutôt qu'à l'aménager. Pour ces raisons, le taux de décrochage scolaire était élevé, malgré les fortes aspirations. Pour bon nombre de Franco-Ontariens, l'obligation de se distancier de ses propres rêves professionnels se traduisait alors en une volonté de devenir anglophone : ne pouvant pas occuper les fonctions qui sont admirées de tous, francophones et anglophones, le francophone deviendra anglophone, il quittera la minorité pour devenir majoritaire. Les analyses avaient aussi révélé que l'instruction modifiait la vision pro-anglaise : les jeunes qui faisaient des études universitaires, notamment, transformaient souvent leur admiration pour l'univers anglais en méfiance et leur mépris pour ce qui est français en reconnaissance. Les analyses avaient de plus montré que les filles parvenaient davantage que les garçons à persister dans le système d'éducation - beaucoup plus en fait que dans d'autres groupes culturels où l'on observe aussi que le décrochage scolaire est plus masculin que féminin -, que cette persévérance avait pour cause une moins grande aliénation des filles à l'anglais et probablement une 
influence différenciée des parents : les mères, instruites ou non, encourageant sans ambages leurs filles à s'instruire, les pères non instruits - et nombreux à l'être - incitant moins systématiquement leurs fils à se scolariser, quoique ces types de relations, bien sûr, n'excluent pas les rapports croisés de la mère et du fils ou du père et de la fille.

Cette interprétation n'a jamais été confirmée au-delà des données dont nous disposions au moment de l'enquête, données qui n'étaient stratifiées qu'en fonction du niveau d'instruction. Elle n'a pas, par exemple, fait l'objet d'une comparaison entre les groupes linguistiques ; on n'a pas démontré si les représentations linguistiques variaient réellement selon la langue, ou même l'ethnie, et, le cas échéant, si elles avaient une incidence sur les aspirations.

Le thème des aspirations a quelque peu été délaissé depuis une quinzaine d'années par les spécialistes des sciences sociales. Il a été supplanté, voire occulté, par celui de l'identité ${ }^{20}$, thème important, s'il en est un, en cette ère de mondialisation où les groupes minoritaires peinent souvent à reproduire les conditions

20. Une bonne partie des réflexions de Joseph-Yvon Thériault vont dans ce sens : L'Identité à l'épreuve de la modernité. Écrits politiques sur l'Acadie et les francophonies canadiennes minoritaires (Moncton, Éditions d'Acadie, 1995) ; Faire société : société civile et espaces francophones (Sudbury, Prise de parole, coll. «Agora », 2007). On le voit dans plusieurs chapitres du livre dirigé par Normand Labrie et Gilles Forlot, L'Enjeu de la langue en Ontario français (Sudbury, Prise de parole, 1999) : celui de Jürgen Erfurt, « Le Changement de l'identité linguistique chez les Franco-Ontariens. Résultat d'une étude de cas » (p. 59-77) ; celui de Laurent Gajo, «Entre immersion et émergence identitaire » (p. 109-128) ; celui de Monica Heller, «Quel(s) français et pour qui ? Discours et pratiques identitaires en milieu scolaire franco-ontarien » (p. 129-165). On le voit aussi dans le livre de Diane Farmer : Artisans de la modernité. Les Centres culturels en Ontario français (Ottawa, Presses de l'Université d'Ottawa, "Amérique française », $\left.\mathrm{n}^{\circ} 4,1996\right)$ et dans celui de Diane Gérin-Lajoie : Parcours identitaires de jeunes francophones en milieu minoritaire (Sudbury, Prise de parole, 2003). On le voit encore dans le chapitre de Fernan Carrière, «La Métamorphose de la communauté franco-ontarienne, 1960-1985 (dans Cornelius J. Jaenen (dir.), Les Franco-Ontariens, Ottawa, Presses de l'Université d'Ottawa, «Ontario Historical Studies Series », 1993, p. 305-340). De manière moins spécifique, on le constate dans deux sections de l'ouvrage dirigé par William Floch et Yves Frenette, Nouvelles perspectives canadiennes (Vitalité des communautés, confiance des communautés. Forum de recherche sur les langues officielles. Analyse et réflexion sur le sondage de GPC International portant sur les attitudes et les perceptions à l'égard des langues officielles, Ministère des Travaux publics et Services gouvernementaux, 
de leur spécificité. Même les sociologues de l'éducation s'en sont quelque peu désintéressés. Mais ce désintéressement ne s'explique certainement pas par le fait que le phénomène ne soit plus utile à la compréhension des populations et de leur avenir, ni non plus parce que toutes les questions qu'il pose ont trouvé des réponses ou que le cas franco-ontarien a été élucidé. Certes, il semble de plus en plus acquis que l'influence de la classe sociale de la famille d'origine soit réelle quoique fragmentaire et qu'il en aille de même des autres facteurs comme le sexe, la race ou l'ethnie ; mais ne peut-on pas concevoir des modélisations plus adéquates qui seront mieux à même de rendre compte d'un objet aussi important ? D'autres questions se posent. On sait que les aspirations des francophones de l'Ontario ont été, dans des recherches antérieures, et de façon récurrente, plus communément élevées que celles des autres groupes ; mais en est-il encore ainsi ? La question est justifiée malgré la récurrence des constats : en effet, si les jeunes formulent des projets de carrière qui sont peu en relation avec les modèles que présentent les parents par l'exercice de leur métier ou avec la structure professionnelle qui s'offre à leur regard quand ils le posent sur leur communauté, c'est sans doute parce que ces modèles transcendent les communautés et donc qu'ils sont transmis par des sources plus communes, comme les médias ; si tel est le cas, on peut s'attendre à ce que la différence entre les francophones et les anglophones s'estompe avec les années, c'està-dire au fur et à mesure que les symboliques collectives s'imposent à celles qui sont issues de la communauté particulière. Dans ces conditions, on devrait même trouver une similitude des représentations linguistiques des francophones et des anglophones; on devrait pareillement découvrir que les aspirations et les représentations des jeunes d'un groupe ethnique autre, celui des Amérindiens par exemple, s'apparentent à celles des jeunes

2005) : celle qui commence par le propos de Daniel Bourgeois, David Bourgeois et Gino Leblanc, « La Confiance des minoritaires et des majoritaires à l'égard de l'épanouissement des communautés de langue officielle » (p. 29-71); celle dont le point de départ est la présentation de Josée Bergeron, « Où est le bilinguisme entre identités et générations ? » (p. 73-100). 
francophones ou anglophones ; il en irait pareillement des variations selon le sexe qui devraient, elles, se dissiper. Si tel n'était pas le cas, cela signifierait que les logiques communautaires auraient préséance sur les forces transculturelles.

\section{II - MÉTHOdologie ET PROFIL DE L'ÉCHANTILLON}

Ce rappel des études sur les aspirations juvéniles en général et en Ontario français permet de soulever quatre grandes questions :

1. Les jeunes franco-ontariens ont-ils toujours plus communément des ambitions plus élevées que les angloontariens?

2. Les ambitions des jeunes d'un autre groupe minoritaire, les Amérindiens notamment, ressemblent-elles à celles des Franco-Ontariens ?

3. Peut-on toujours soutenir qu'une différenciation des aspirations entre les francophones et les anglophones, si elle se donnait à l'observation, pourrait s'expliquer par recours à un sentiment de minoritude ? Si les aspirations ne distinguaient pas les groupes, les représentations relatives à la culture pourraient-elles rendre compte de la variation des opinions selon les individus?

4. Les déterminants des aspirations juvéniles qu'évoque la sociologie traditionnelle sont sans doute relatifs, mais peuton en faire intervenir d'autres?

Pour répondre à ces questions, nous disposons des données d'une enquête qui a été menée dans le nord-est de l'Ontario, dans la région desservie par la Commission de formation du Nord-Est, soit, en gros, de la Baie de James à Timmins et de Hearst au Témiscamingue ${ }^{21}$. Un questionnaire a été distribué dans les classes de neuvième et de douzième années de dix-sept écoles secondaires au printemps 2005 - il est prévu que les élèves qui ont répondu au questionnaire seront sondés annuellement jusqu'en 2014.

21. Pour de plus amples informations sur cette enquête, on pourra lire : Simon Laflamme et Pierre Bouchard, Les Jeunes et le Nord. Un parcours à découvrir, Hearst, Commission de formation du Nord-Est, 2005, 184 p. On peut lire ce rapport en version électronique sur le site de la Commission : http://www.fnetb.com/French/Youth/ LesjeunesetleNordabreg.pdf (version abrégée : http://www.fnetbcfne.on.ca/pdf/ Rapports/Les\%20jeunes\%20et\%20le\%20Nord\%20abrege\%20final.pdf). 
Quelques écoles n'ont pas participé à cette recherche, et un certain nombre d'élèves n'ont pas pu répondre au questionnaire; nous traiterons donc cet ensemble comme un échantillon - et non comme s'il s'agissait de cette population elle-même - des deux niveaux visés pour la région du Nord-Est, même si une forte proportion des élèves ont collaboré à la recherche. Ces données peuvent aussi être traitées comme une grappe échantillonnale de l'Ontario pourvu que l'extrapolation se fasse avec réserve, que soit pris en considération, entre autres, le fait que la région étudiée est moins ethniquement composite que le Centre-Sud ou l'Est de la province.

L'échantillon compte 1758 individus, dont 936 en neuvième année et 788 en douzième année (34 élèves n'ayant pas fourni l'information). Dans cet ensemble, on dénombre 846 filles et 907 garçons (dans cinq cas l'information n'est pas disponible). Les proportions des deux sexes sont très semblables aux deux niveaux examinés : $48,1 \%$ et $48,7 \%$ pour les filles en neuvième et en douzième, et donc $51,9 \%$ et $50,3 \%$ respectivement pour les garçons. La distribution des élèves selon la langue d'enseignement traduit bien la situation de la région : 28,2 \% des jeunes sont inscrits dans des écoles françaises, 38,3\% dans des écoles anglaises, 27,1\% dans des établissements bilingues ; 6,4\% suivent leurs cours dans des programmes anglais qui comprennent une forte composante française. Si l'on demande à l'élève « dans quelle langue suis-tu la plupart de tes cours ? », $62 \%$ répondent « en anglais » et $38 \%$, « en français ». La langue maternelle est le français seulement pour $21,9 \%$ des individus de l'échantillon, l'anglais seulement pour 39,1\% ; pas moins de 34,2\% des jeunes déclarent pour langues maternelles et le français et l'anglais; les $4,8 \%$ restant présentent d'autres langues maternelles ou diverses combinaisons. Si l'on demande aux jeunes « parmi ces groupes, auquel t'identifies-tu le plus? », et donc qu'on limite les possibilités, on obtient les réponses suivantes: Premières Nations, $6,9 \%$; francophones, 35,8 \% ; anglophones, 52,9 \% ; autres, $4,5 \%$. 


\section{III - DES ASPIRATIONS COMPARABLES}

Le principal questionnement de notre démarche a trait à la variation des aspirations.

\section{A. Les aspirations éducationnelles}

Pour observer ces orientations sur le plan éducationnel, nous avons posé la question suivante : «Quel niveau d'instruction auras-tu atteint quand tu auras terminé toutes tes études? » L'élève pouvait alors choisir parmi cinq réponses :

1. quelques années de l'école secondaire

2. diplôme d'études secondaires

3. diplôme d'études collégiales

4. diplôme d'études universitaires de $1^{\text {er }}$ cycle (B.A., B.Sc., B.Éd....)

5. diplôme d'études universitaires de niveau supérieur (maîtrise, doctorat)

Les réponses qui sont fournies peuvent être mises en relation avec diverses variables ethnolinguistiques. On peut vérifier si ces intentions dépendent de la langue de l'établissement scolaire. En prenant la langue dans laquelle l'élève suit la plupart de ses cours, on note une différence selon qu'il s'agit du français ou de l'anglais. Elle se manifeste surtout chez les jeunes qui ne prévoient pas s'instruire après les études secondaires et chez ceux qui convoitent les études supérieures (voir le Tableau 1) : dans le premier cas, les études en français paraissent moins souvent freiner l'inclination à faire des études postsecondaires; dans le second cas, elles semblent davantage laisser miroiter les plus hautes études. Il faut cependant noter que les variations, bien que consistantes, ne sont pas très marquées; il faut aussi signaler que les chiffres pour les études collégiales et pour le diplôme de premier cycle universitaire s'avèrent tout à fait comparables. Être minoritaire francophone en Ontario, ce n'est certainement pas être empêché de se projeter dans les études postsecondaires comme le fait le majoritaire.

Si l'on examine la distribution des projets d'études en fonction, cette fois, de la langue maternelle, les chiffres vont dans 


\section{Tableau 1}

Niveau de scolarité prisé

selon la langue dans laquelle l'élève suit la plupart de ses cours

(Distribution en pourcentage)

\begin{tabular}{|l|r|r|}
\hline Niveau de scolarité prisé & \multicolumn{2}{|c|}{$\begin{array}{l}\text { Langue dans laquelle } \\
\text { l'élève suit la plupart } \\
\text { de ses cours }\end{array}$} \\
& français & anglais \\
\hline Quelques années de l'école secondaire & 0,7 & 2,4 \\
\hline Diplôme d'études secondaires & 5,9 & 14,9 \\
\hline Diplôme d'études collégiales & 41,5 & 37,4 \\
\hline Diplôme d'études universitaires de 1 ${ }^{\text {er }}$ cycle & 29,8 & 29,6 \\
\hline Diplôme d'études universitaires de niveau supérieur & 22,1 & 15,7 \\
\hline $\begin{array}{l}\text { Total } \\
(\mathrm{n})\end{array}$ & $\begin{array}{c}100,0 \\
(578)\end{array}$ & 100,0 \\
\hline$\chi_{(4)}^{2}=40,95 ; \mathrm{p}_{2}<0,001$ & \\
\hline
\end{tabular}

le même sens (voir le Tableau 2) : un peu plus de francophones que d'anglophones sont attirés par les études supérieures; un peu plus d'anglophones que de francophones ne souhaitent pas s'inscrire dans un collège ou une université ; à peu près autant de Français que d'Anglais prévoient qu'ils auront obtenu un diplôme collégial ou universitaire. Les jeunes qui se déclarent à la fois Français et Anglais se situent entre les deux premiers groupes pour ce qui est des plus grands lieux de différence, le diplôme d'études secondaires seulement ou le diplôme d'études supérieures; ils sont un peu plus nombreux à entrevoir le diplôme d'études collégiales, un peu moins, le diplôme de premier cycle universitaire. On notera, par ailleurs, la particularité de la distribution des pourcentages chez les autres groupes linguistiques : si une langue autre que le français et l'anglais ou une autre combinaison de langues maternelles accroît la résistance envers les études postsecondaires, elle augmente par contre la propension à faire des études supérieures.

Si l'on décante ces pourcentages en fonction de l'identité (voir le Tableau 3), la variation entre francophones et anglophones 


\section{Tableau 2}

Niveau de scolarité prisé selon la (les) langue(s) maternelle(s)

(Distribution en pourcentage)

\begin{tabular}{|c|c|c|c|c|}
\hline \multirow[t]{2}{*}{ Niveau de scolarité prisé } & \multicolumn{4}{|c|}{ Langue(s) maternelle(s) } \\
\hline & français & anglais & $\begin{array}{c}\text { français } \\
\text { et } \\
\text { anglais }\end{array}$ & autres \\
\hline Quelques années de l'école secondaire & 1,2 & 2,4 & 0,6 & 5,6 \\
\hline Diplôme d'études secondaires & 4,6 & 15,9 & 9,8 & 16,8 \\
\hline Diplôme d'études collégiales & 38,3 & 36,6 & 44,6 & 27,1 \\
\hline Diplôme d'études universitaires de $1^{\text {er }}$ cycle & 32,8 & 30,7 & 27,2 & 24,3 \\
\hline $\begin{array}{l}\text { Diplôme d'études universitaires } \\
\text { de niveau supérieur }\end{array}$ & 23,0 & 14,5 & 17,8 & 26,2 \\
\hline $\begin{array}{l}\text { Total } \\
(\mathrm{n})\end{array}$ & $\begin{array}{l}100,0 \\
(326)\end{array}$ & $\begin{array}{l}100,0 \\
(593)\end{array}$ & $\begin{array}{l}100,0 \\
(500)\end{array}$ & $\begin{array}{l}100,0 \\
(107)\end{array}$ \\
\hline \multicolumn{5}{|c|}{$\begin{array}{l}\chi_{(12)}^{2}=66,12 ; p<0,001 \\
\text { La somme des colonnes ne donne pas toujours exactement } 100,0 \% \text { à cause } \\
\text { de la règle qui a été suivie pour arrondir les décimales. }\end{array}$} \\
\hline
\end{tabular}

s'atténue ; il n'y a plus que 6,4 \% qui séparent les anglophones des francophones pour ce qui est de l'aspiration au diplôme d'études secondaires, $3 \%$ qui éloignent ceux-là de ceux-ci pour ce qui est du désir d'obtenir un diplôme d'études supérieures. On trouve plus de francophones (479) qu'on avait de personnes de langue maternelle française (326) de même que plus d'anglophones (739) qu' on avait de jeunes de langue maternelle anglaise (593). Le groupe autre témoigne à nouveau d'une attirance marquée pour les études supérieures, mais avec un chiffre qui n'est que légèrement supérieur à celui qu'affichent les francophones. L'apparition du groupe des Premières Nations fait surgir des écarts importants avec les trois autres ensembles : plus de jeunes qui ne veulent pas faire d'études postsecondaires, moins qui éprouvent quelque attrait pour les études universitaires.

\section{B. Les aspirations professionnelles}

Pour étudier les aspirations professionnelles, nous avons posé la question : « Cinq ans après la fin de tes études, quel genre d'emploi 


\section{Tableau 3}

Niveau de scolarité prisé selon le groupe auquel le jeune s'identifie le plus (Distribution en pourcentage)

\begin{tabular}{|l|r|r|r|r|}
\hline Niveau de scolarité prisé & \multicolumn{3}{|c|}{$\begin{array}{c}\text { Groupe auquel le jeune } \\
\text { s'identifie le plus }\end{array}$} \\
& $\begin{array}{r}\text { Premières } \\
\text { Nations }\end{array}$ & $\begin{array}{r}\text { Franco- } \\
\text { phones }\end{array}$ & $\begin{array}{r}\text { Anglo- } \\
\text { phones }\end{array}$ & Autres \\
\hline Quelques années de l'école secondaire & 10,1 & 0,4 & 1,4 & 3,8 \\
\hline Diplôme d'études secondaires & 21,3 & 6,5 & 12,9 & 9,4 \\
\hline Diplôme d'études collégiales & 47,2 & 42,0 & 36,4 & 35,8 \\
\hline $\begin{array}{l}\text { Diplôme d'études universitaires } \\
\text { de 1 er cycle }\end{array}$ & 15,7 & 30,1 & 31,3 & 26,4 \\
\hline $\begin{array}{l}\text { Diplôme d'études universitaires de } \\
\text { niveau supérieur }\end{array}$ & 5,6 & 21,1 & 18,1 & 24,5 \\
\hline $\begin{array}{l}\text { Total } \\
\text { (n) }\end{array}$ & 100,0 & 100,0 & 100,0 & 100,0 \\
& $(89)$ & $(479)$ & $(739)$ & $(53)$ \\
\hline $\begin{array}{l}\chi_{(12)}^{2}=85,18 ; p<0,001 \\
\text { La somme des colonnes ne donne pas toujours exactement 100,0 \% à cause } \\
\text { de la règle qui a été suivie pour arrondir les décimales. }\end{array}$ \\
\hline
\end{tabular}

auras-tu ? » Les jeunes ont fourni une réponse dans un espace qui ne comportait aucune catégorie prédéfinie. Ces réponses ont ensuite été classées à l'aide du système établi par le ministère de l'Industrie du Canada ${ }^{22}$. Nous avons attribué à chacune des classes du système une cote d'un à cinq afin de les hiérarchiser puis de rendre possible un traitement cardinal de la variable. Pour nous aider dans cette opération, nous avons pris en considération le revenu moyen et le niveau d'instruction médian de chacun des postes qui figurent dans les classes du système de même que le degré de prestige qui peut être associé à ces titres ${ }^{23}$. Suivant une dénomination connue, les cinq valeurs de la variable profession sont les suivantes :

22. Classification type des professions (1991), Ottawa, Ministère de l'Industrie, des Sciences et de la Technologie, 12-565F au catalogue, 1993.

23. Pour établir le niveau de prestige, nous avons présenté des professions à 539 jeunes. Ils ont indiqué sur une échelle allant de 1 à 100 la valeur qu'ils accordaient à chacune d'elles. La valeur moyenne pour chacune des professions a compté pour $10 \%$ dans le calcul du niveau de la profession; l'instruction et le revenu, pour $45 \%$ chacun. 
1. inférieur

2. moyen-inférieur

3. moyen

4. moyen-supérieur

5. supérieur

On trouve, par exemple, dans le premier niveau, des titres, ainsi que les jeunes eux-mêmes les formulent, comme coiffeur, menuisier, serveur dans un restaurant, tatoueur, vendeur; dans le deuxième, comme bûcheron, chef cuisinier, dessinateur d'intérieur, électricien, mineur, photographe, soudeur ; dans le troisième, comme écrivain, infirmier, journaliste, physiothérapeute, policier, technicien en informatique ; dans le quatrième, comme directeur du personnel, enseignant (au primaire ou au secondaire), pilote de ligne, pharmacien, programmeur, travailleur social ; dans le cinquième, enfin, comme avocat, biologiste, dentiste, ingénieur civil, médecin, propriétaire de grande entreprise, vétérinaire.

En fonction de la langue dans laquelle l'élève suit la majorité de ses cours, il n'y a pas de différence de moyennes qui soit significative ; si cette langue est le français, la moyenne est de $3,07(\mathrm{~s}=1,40)$, si elle est l'anglais, elle est de $2,92(\mathrm{~s}=1,41)^{24}$. On peut se demander si cette analyse sur les moyennes cache des informations importantes. Pour le vérifier, il suffit d'examiner les liens entre chacune des langues et les divers niveaux de professions. On obtient alors un tableau de contingence dont les variations doivent être attribuées au hasard ${ }^{25}$. À titre d'illustration, dans ce tri à plat, on lit que, parmi les jeunes dont la langue la plus commune d'enseignement est le français, il y en a $18,9 \%$ qui se destinent aux professions de niveau supérieur ; chez ceux qui étudient principalement en anglais, ce pourcentage est tout à fait comparable, à 17,2\%.

Le recours à la langue maternelle ne permet pas non plus de découvrir de réelle inégalité de moyennes ; les différences ne

24. $\mathrm{t}_{(1186)}=1,77 ; \mathrm{p}=0,08$.

25. $\chi_{(4)}^{2}=8,17 ; \mathrm{p}=0,09$. 
sont pas attribuables au hasard ${ }^{26}$, mais les moyennes sont très proches les unes des autres : français, 3,19 $(\mathrm{s}=1,34)$; anglais, $2,83(\mathrm{~s}=1,40)$; français et anglais, 3,00 $(\mathrm{s}=1,42)$; autre, 2,98 $(s=1,53)$. Il annonce des variations entre les pourcentages ${ }^{27}$, mais rien qui soit susceptible de retenir l'attention. Il y a un certain ascendant pour les francophones par comparaison aux anglophones, mais tout en retenue. L'attrait pour les professions les plus élevées, en guise d'illustration, est de $18,1 \%$ pour les premiers, de $14,9 \%$ pour les deuxièmes, de $20,0 \%$ pour les bilingues et de $22,0 \%$ pour les autres.

L'analyse d'après le groupe auquel le jeune s'identifie met bien en évidence cette proximité entre francophones et anglophones ; les moyennes sont alors de 3,08 $(\mathrm{s}=1,38)$ pour les premiers et de 3,02 $(\mathrm{s}=1,42)$ pour les seconds. Cette analyse rappelle également un certain décalage pour les jeunes des Premières Nations dont la moyenne est de 2,37 $(\mathrm{s}=1,16)$. Pour ceux qui appartiennent à l'ensemble « autres », la moyenne 2,74 $(\mathrm{s}=1,43)$ se situe entre celle des élèves qui s'identifient aux Premières Nations, d'une part, et les francophones et les anglophones, d'autre part ${ }^{28}$. Le tableau de contingence permet de bien percevoir ces similitudes et ces différences (voir le Tableau 4). Les distributions pour les francophones et les anglophones sont similaires. L'appel pour les professions de niveau supérieur a lieu dans des proportions semblables pour tout le monde, sauf pour les jeunes Amérindiens où il est moins fréquent. Les professions de niveau moyen-supérieur présentent plus d'attrait pour les francophones et les anglophones que pour les jeunes des deux autres groupes ; inversement, les professions de niveau moyen sont moins souvent mentionnées par les francophones et les anglophones que par les élèves des deux autres ensembles.

26. $\mathrm{F}_{(3: 1194)}=3,72 ; \mathrm{p}<0,05$.

27. $\chi_{(12)}^{(3 ; 194)}=25,94 ; \mathrm{p}<0,05$.

28. $\mathrm{F}_{(3 ; 1063)}=5,63 ; \mathrm{p}<0,01$. 


\section{Tableau 4}

Niveau de la profession prisée selon le groupe auquel le jeune s'identifie le plus

(Distribution en pourcentage)

\begin{tabular}{|c|c|c|c|c|}
\hline \multirow[t]{2}{*}{ Niveau de la profession prisée } & \multicolumn{4}{|c|}{$\begin{array}{l}\text { Groupe auquel le jeune } \\
\text { s'identifie le plus }\end{array}$} \\
\hline & $\begin{array}{c}\text { Premières } \\
\text { Nations }\end{array}$ & $\begin{array}{l}\text { Franco- } \\
\text { phones }\end{array}$ & $\begin{array}{c}\text { Anglo- } \\
\text { phones }\end{array}$ & Autres \\
\hline Inférieur & 27,9 & 18,3 & 21,6 & 26,3 \\
\hline Moyen-inférieur & 27,9 & 18,0 & 16,0 & 18,4 \\
\hline Moyen & 29,4 & 19,5 & 20,9 & 28,9 \\
\hline Moyen-supérieur & 8,8 & 25,4 & 22,3 & 7,9 \\
\hline Supérieur & 5,9 & 18,8 & 19,2 & 18,4 \\
\hline $\begin{array}{l}\text { Total } \\
\text { (n) }\end{array}$ & $\begin{array}{r}100,0 \\
(68)\end{array}$ & $\begin{array}{l}100,0 \\
(405)\end{array}$ & $\begin{array}{l}100,0 \\
(556)\end{array}$ & $\begin{array}{r}100,0 \\
(38)\end{array}$ \\
\hline
\end{tabular}

\section{Parenthèses sur les aspirations professionnelles}

Il nous semble important d'attirer l'attention sur trois points qui sautent aux yeux quand on se penche sur les projets de carrière.

D'abord, près de $14 \%$ des filles et $25 \%$ des garçons ne sont pas en mesure de se projeter dans un emploi spécifique, ni même dans un domaine général. Cette incapacité touche semblablement les jeunes de langue maternelle française (23\%) ou anglaise $(27 \%)$; elle a trait à une proportion semblable (22\%) des élèves qui déclarent pour langues maternelles le français et l'anglais à la fois. Elle concerne $41 \%$ de ceux qui se disent de langue maternelle autre ou d'une autre combinaison de langues. Cela ne pose pas de problème en soi ; cela traduit même une certaine sagacité en ce qu'il y a là le témoignage d'une ouverture pour ce qui peut se dessiner au cours des années. 
Ensuite, on remarque une forte récurrence de certaines professions : $37 \%$ de tous les répondants choisissent leur emploi à l'intérieur de 22 possibilités. Pas moins de 146 jeunes veulent enseigner à l'élémentaire ou au secondaire ; 78 se voient comme médecins, généralistes ou spécialistes ; 68 s'orientent vers les fonctions de policier ; 53 occuperaient des postes de mécanicien ; 34 seraient ingénieurs ; 33 prévoient être avocats. Vingthuit autres songent à être infirmiers, 25 à être comptables, 25 à être techniciens en informatique; il y aurait aussi 23 électriciens, 23 vétérinaires, 21 coiffeurs, 21 soudeurs, 18 psychologues, 17 ouvriers de la construction, 13 travailleurs sociaux, 11 artistes et 10 hygiénistes dentaires.

Enfin, on note une forte répartition des professions selon le sexe. Parmi les 34 ingénieurs, il n'y aura que 3 femmes; les 25 techniciens en informatique seront tous des hommes; parmi les infirmiers, il n'y aura que 2 hommes ; toutes les hygiénistes dentaires seront des femmes ; parmi les 146 enseignants, 102 seront des femmes ; il n'y aura pas d'homme parmi les coiffeurs, pas de femme parmi les ouvriers de la construction ; il n'y en aura qu'une seule parmi les mécaniciens, une seule parmi les électriciens, que trois parmi les soudeurs; il y aura deux fois plus de policiers que de policières ; les comptables seront en majorité des hommes (16/25). Une nuance à apporter à cette division stéréotypée des sexes : les femmes seront majoritaires parmi les médecins (43/78), les vétérinaires (18/23), les avocats (20/33), les psychologues $(15 / 18)$ et les travailleurs sociaux $(10 / 13)$.

\section{IV - AsPirations et REPRÉSENTATIONS LINGUISTIQUeS}

On trouve peu de variations entre les groupes, ni d'après la langue, ni selon la population à laquelle les jeunes s'identifient; mais la différence entre les individus à l'intérieur de ces groupes peutelle s'expliquer en fonction de la manière dont ils perçoivent leur univers sur le plan culturel ? 
Pour faire cette observation, nous disposons de douze variables $^{29}$ :

1. Il m'arrive de me sentir mal à l'aise quand je m'exprime en anglais

2. Dans le monde, les pays qui ne sont pas anglophones ont peu d'influence

3. On ne peut pas faire grand-chose dans le monde si on ne parle pas l'anglais

4. La diversité culturelle est une richesse pour un pays

5. Dans tous les pays, la science se fait en anglais

6. Le français en Ontario est en voie de disparition

7. La diversité culturelle est une richesse pour le monde

8. Avec la mondialisation, les humains sont de plus en plus différents

9. Presque tout le cinéma du monde est en anglais

10. Avec la mondialisation, les humains sont de plus en plus semblables

11. Il m'arrive de me sentir mal à l'aise quand je m'exprime en français

12. Le français dans le monde est en voie de disparition

Chacune de ces variables est un énoncé à côté duquel l'élève trouve une échelle de « $1 »$, qui signifie « pas du tout d'accord», à « 6 », qui signifie « tout à fait d'accord », où il est par conséquent invité à prendre position.

On peut se demander si ces positions varient selon le groupe auquel le jeune dit s'identifier (voir le Tableau 5). Ce qui se manifeste avant tout quand on répartit les moyennes, c'est leur similitude : il y a peu de différences entre les groupes, même quand l'inégalité est inférable ; les groupes ne prennent pas normalement des positions tranchées ; à l'intérieur de chacun des ensembles, les variations individuelles sont visibles, les écarts types étant toujours supérieurs à 1,22. Cela étant dit, certaines observations ne sont pas sans importance. Une première a trait à une certaine aliénation des francophones : ce sont les anglophones

29. Ces variables ont été utilisées dans l'enquête qui a conduit à la rédaction de L'Ambition démesurée, op. cit. Elles rendront possibles des comparaisons dans l'enquête longitudinale entre les années de même qu'entre l'enquête de L'Ambition démesurée et l'enquête longitudinale. 


\section{Tableau 5}

Différence de moyennes $(\bar{x})$

pour divers énoncés se rapportant aux représentations culturelles

selon le groupe auquel le jeune s'identifie le plus.

Analyses de variances

(Le degré de liberté minimum pour les individus est de 1221)

( $1=$ pas du tout d'accord ; 6 = tout à fait d'accord)

\begin{tabular}{|c|c|c|c|c|c|c|c|}
\hline \multirow{2}{*}{\multicolumn{2}{|c|}{ Énoncé }} & \multicolumn{4}{|c|}{$\begin{array}{l}\text { Groupe auquel le jeune } \\
\text { s'identifie le plus }\end{array}$} & & \multirow[t]{2}{*}{$\mathrm{p}<0,05$} \\
\hline & & $\begin{array}{c}\text { Premières } \\
\text { Nations }\end{array}$ & $\begin{array}{l}\text { Franco- } \\
\text { phones }\end{array}$ & $\begin{array}{l}\text { Anglo- } \\
\text { phones }\end{array}$ & Autres & & \\
\hline $\begin{array}{l}\text { Il m'arrive de me sentir mal à l'aise } \\
\text { quand je m'exprime en anglais }\end{array}$ & $\begin{array}{c}\overline{\mathrm{x}} \\
(\mathrm{s})\end{array}$ & $\begin{array}{r}2,60 \\
(1,74) \\
\end{array}$ & $\begin{array}{r}2,93 \\
(1,67)\end{array}$ & $\begin{array}{r}1,91 \\
(1,55)\end{array}$ & $\begin{array}{r}2,39 \\
(1,73)\end{array}$ & 40,58 & Oui \\
\hline $\begin{array}{l}\text { Dans le monde, les pays qui ne sont } \\
\text { pas anglophones ont peu d'influence }\end{array}$ & $\begin{array}{c}\overline{\mathrm{x}} \\
(\mathrm{s})\end{array}$ & $\begin{array}{r}2,86 \\
(1,34)\end{array}$ & $\begin{array}{r}2,75 \\
(1,47)\end{array}$ & $\begin{array}{r}2,51 \\
(1,54)\end{array}$ & $\begin{array}{r}2,33 \\
(1,45)\end{array}$ & 3,84 & Oui \\
\hline $\begin{array}{l}\text { On ne peut pas faire grand-chose dans } \\
\text { le monde si on ne parle pas l'anglais }\end{array}$ & $\begin{array}{c}\bar{x} \\
(\mathrm{~s})\end{array}$ & $\begin{array}{r}3,23 \\
(1,57)\end{array}$ & $\begin{array}{r}3,63 \\
(1,63)\end{array}$ & $\begin{array}{r}2,86 \\
(1,59)\end{array}$ & $\begin{array}{r}3,14 \\
(1,74)\end{array}$ & 21,68 & Oui \\
\hline $\begin{array}{l}\text { La diversité culturelle est une } \\
\text { richesse pour un pays }\end{array}$ & \begin{tabular}{|c|}
$\bar{x}$ \\
$(\mathrm{~s})$
\end{tabular} & $\begin{array}{r}3,40 \\
(1,42)\end{array}$ & $\begin{array}{r}3,88 \\
(1,46) \\
\end{array}$ & $\begin{array}{r}3,73 \\
(1,57)\end{array}$ & $\begin{array}{r}3,66 \\
(1,84)\end{array}$ & 2,57 & Non \\
\hline $\begin{array}{l}\text { Dans tous les pays, la science } \\
\text { se fait en anglais }\end{array}$ & $\begin{array}{l}\overline{\mathrm{X}} \\
(\mathrm{s})\end{array}$ & $\begin{array}{r}2,88 \\
(1,47)\end{array}$ & $\begin{array}{r}2,55 \\
(1,40)\end{array}$ & $\begin{array}{r}2,55 \\
(1,50)\end{array}$ & $\begin{array}{r}2,45 \\
(1,35)\end{array}$ & 1,35 & Non \\
\hline $\begin{array}{l}\text { Le français en Ontario est } \\
\text { en voie de disparition }\end{array}$ & \begin{tabular}{|l|}
$\bar{x}$ \\
$(s)$
\end{tabular} & $\begin{array}{r}2,55 \\
(1,35)\end{array}$ & $\begin{array}{r}3,48 \\
(1,52)\end{array}$ & $\begin{array}{r}2,67 \\
(1,54) \\
\end{array}$ & $\begin{array}{r}3,00 \\
(1,71) \\
\end{array}$ & 28,07 & Oui \\
\hline $\begin{array}{l}\text { La diversité culturelle est } \\
\text { une richesse pour le monde }\end{array}$ & $\begin{array}{l}\overline{\mathrm{x}} \\
(\mathrm{s})\end{array}$ & $\begin{array}{r}3,27 \\
(1,42)\end{array}$ & $\begin{array}{r}3,90 \\
(1,52)\end{array}$ & $\begin{array}{r}3,86 \\
(1,61)\end{array}$ & $\begin{array}{r}3,83 \\
(1,87)\end{array}$ & 3,57 & Oui \\
\hline $\begin{array}{l}\text { Avec la mondialisation, les humains } \\
\text { sont de plus en plus différents }\end{array}$ & $\begin{array}{c}\overline{\mathrm{x}} \\
(\mathrm{s})\end{array}$ & $\begin{array}{r}3,37 \\
(1,44)\end{array}$ & $\begin{array}{r}3,70 \\
(1,39) \\
\end{array}$ & $\begin{array}{r}3,46 \\
(1,46)\end{array}$ & $\begin{array}{r}3,67 \\
(1,38)\end{array}$ & 3,04 & Oui \\
\hline $\begin{array}{l}\text { Presque tout le cinéma du monde } \\
\text { est en anglais }\end{array}$ & $\begin{array}{l}\bar{x} \\
(s)\end{array}$ & $\begin{array}{r}3,34 \\
(1,51)\end{array}$ & $\begin{array}{r}3,87 \\
(1,53)\end{array}$ & $\begin{array}{r}3,16 \\
(1,53)\end{array}$ & $\begin{array}{r}3,21 \\
(1,58)\end{array}$ & 20,06 & Oui \\
\hline $\begin{array}{l}\text { Avec la mondialisation, les humains } \\
\text { sont de plus en plus semblables }\end{array}$ & $\begin{array}{c}\overline{\mathrm{x}} \\
(\mathrm{s})\end{array}$ & $\begin{array}{r}3,04 \\
(1,44)\end{array}$ & $\begin{array}{r}2,96 \\
(1,36)\end{array}$ & $\begin{array}{r}3,05 \\
(1,47)\end{array}$ & $\begin{array}{r}3,58 \\
(1,57)\end{array}$ & 2,78 & Oui \\
\hline $\begin{array}{l}\text { Il m'arrive de me sentir mal à l'aise } \\
\text { quand je m'exprime en français }\end{array}$ & \begin{tabular}{l|}
$\bar{x}$ \\
$(s)$
\end{tabular} & $\begin{array}{r}3,66 \\
(1,71) \\
\end{array}$ & $\begin{array}{r}2,30 \\
(1,57)\end{array}$ & $\begin{array}{r}3,69 \\
(1,65)\end{array}$ & $\begin{array}{r}3,43 \\
(1,80)\end{array}$ & 67,90 & Oui \\
\hline $\begin{array}{l}\text { Le français dans le monde est } \\
\text { en voie de disparition }\end{array}$ & $\begin{array}{l}\overline{\mathrm{x}} \\
(\mathrm{s})\end{array}$ & $\begin{array}{r}2,55 \\
(1,22)\end{array}$ & $\begin{array}{r}3,22 \\
(1,54)\end{array}$ & $\begin{array}{r}2,63 \\
(1,51)\end{array}$ & $\begin{array}{r}2,92 \\
(1,60)\end{array}$ & 14,71 & Oui \\
\hline
\end{tabular}

qui sont le moins en accord $(\overline{\mathrm{x}}=2,86)$ avec la proposition selon laquelle « on ne peut pas faire grand-chose dans le monde si on ne parle pas l'anglais ", c'est chez les francophones que l'assentiment est le plus marqué $(\bar{x}=3,63)$; ce sont les volume 82010 
francophones, avec une moyenne de 3,48, qui inclinent le plus à croire que « le français en Ontario est en voie de disparition », les anglophones ne présentant une moyenne que de 2,67; on fait un constat semblable pour l'éventualité de la disparition du français dans le monde; on peut ajouter que ce sont encore les francophones qui s'imaginent le plus $(\overline{\mathrm{x}}=3,87)$ que « presque tout le cinéma du monde est en anglais » (la moyenne pour les anglophones étant de 3,16 ). Une deuxième observation concerne les deux énoncés sur les bienfaits de la diversité culturelle : les moyennes n'atteignent pas la valeur de quatre et les écarts types signalent des variations notoires entre les individus, ce qui traduit donc une certaine hésitation pour plusieurs à admettre que cette diversité culturelle représente une richesse, pour un pays ou pour le monde.

Pour vérifier l'influence de ces énoncés sur les aspirations professionnelles, on peut effectuer une analyse de régression multiple et repérer lesquels parmi eux ont quelque incidence (méthode stepwise dans le logiciel SPSS). On peut le faire pour tout l'échantillon. Deux énoncés sont alors retenus : « la diversité culturelle est une richesse pour un pays », le coefficient est positif $(ß=0,16)$, et « il m'arrive de me sentir mal à l'aise quand je m'exprime en anglais », le coefficient est négatif $(\beta=-0,08)$. Plus on est favorable à l'idée qui veut que la diversité culturelle soit bénéfique et moins on tend à être gêné quand on parle en anglais, alors plus est élevé le statut de la profession qu'on envisage. Ensemble, ces deux énoncés toutefois n'expliquent que $3 \%$ de la variance des plans des élèves ${ }^{30}$. Si l'on répète la même analyse pour les personnes qui s'identifient comme francophones seulement, une seule proposition est sélectionnée et c'est à nouveau celle qui porte sur la richesse de la diversité culturelle dans un pays. Le coefficient est encore positif $(\beta=0,15)$; la variance expliquée est à nouveau minime $\left(\mathrm{R}^{2}=2 \%\right)^{31}$. Si l' analyse ne s'intéresse maintenant qu'aux anglophones, elle retient trois

30. $\mathrm{F}=13,13 ; \mathrm{p}<0,001$.

31. $\mathrm{F}=5,81 ; \mathrm{p}<0,05$. 
énoncés : « la diversité culturelle est une richesse pour un pays », « il m'arrive de me sentir mal à l'aise quand je m'exprime en français » et « il m'arrive de me sentir mal à l'aise quand je m'exprime en anglais »; les coefficients des deux premières propositions sont positifs ( $\beta=0,13$ et 0,12 respectivement) et celui de la troisième est négatif $(\beta=-0,11)$; la variance expliquée par ces trois énoncés n'est que de $5 \%{ }^{32}$. Si elle se rapporte aux Amérindiens, un nouvel énoncé apparaît : « on ne peut pas faire grand-chose dans le monde si l'on ne parle pas l'anglais »; le coefficient est positif $(\beta=0,36)$ : plus on le pense, plus les aspirations professionnelles sont élevées ; la variance expliquée atteint tout de même $13 \%{ }^{33}$. Aucune proposition n'est retenue dans un échantillon qui ne comporte que les jeunes qui ne s'identifient ni aux Premières Nations, ni aux anglophones, ni aux francophones.

\section{V - D'AUTRES DÉTERMINANTS DES ASPIRATIONS PROFESSIONNELLES}

L'enquête que nous avons menée a permis de recueillir de nombreuses informations. Parmi elles, on compte, il va sans dire, le sexe du répondant, la scolarité et la profession de chacun des parents des élèves. Il y a aussi une série de seize énoncés sur la façon de concevoir la famille ; à titre d'exemple, on y lit des assertions comme « plus tard, je me marierai », « il vaut mieux avoir un emploi stable avant d'avoir des enfants » ou « la relation entre moi et ma conjointe sera harmonieuse ». Une autre série mentionne 31 activités comme « je lis des journaux imprimés », « je vais au cinéma », « je vais à la pêche ». Une autre ensuite indique 29 usages d'Internet comme « j'envoie des messages électroniques en dehors du cadre de mes études », «j'utilise Internet pour acheter des produits », « j'utilise Internet pour écouter la radio ». Une autre encore propose 42 pensées sur divers sujets ; c'est là qu'apparaissent les énoncés sur la langue et la culture ; à côté de ces énoncés, on lit aussi des formulations

32. $\mathrm{F}=7,42 ; \mathrm{p}<0,001$.

33. $\mathrm{F}=6,08 ; \mathrm{p}<0,05$. 
relatives à divers sujets comme « les études collégiales sont mieux orientées vers l'emploi que les études universitaires », « ma communauté a suffisamment de magasins », « je m'intéresse aux questions politiques dans le monde », « ma relation avec mes enseignants est harmonieuse ». Huit autres énoncés se rapportent à la santé ; par exemple « comparativement à d'autres personnes de mon âge, ma santé physique est... » ou « au cours des trois derniers mois, je me suis senti-e désespéré-e en pensant à l'avenir ». Tous ces énoncés correspondent à une échelle à six niveaux où les valeurs oscillent entre « pas du tout d'accord » et " tout à fait d'accord », entre " jamais » et " très souvent » ou entre « mauvaise » et « excellen-te ». Ils constituent donc tous des variables cardinales. Nous avons transformé la variable sexe de telle sorte qu'elle devienne, de nominale qu'elle est, cardinale, en attribuant aux garçons la valeur « 0 » et aux filles la valeur « 1 ». Nous avons ainsi pu disposer de 137 variables exogènes dont nous avons testé la contribution à l'explication de la variance de l'aspiration professionnelle. Pour ce faire, nous avons recouru à une analyse de régression multiple qui sélectionne par tâtonnement (méthode stepwise dans le logiciel SPSS) les variables dont l'influence est significative. Les résultats que nous avons obtenus sont parfois étonnants, ils sont la plupart du temps instructifs (voir le Tableau 6).

Avec toutes ces variables, en faisant l'analyse, dans bien des cas, sans raison théorique évidente, donc en donnant au modèle toutes les chances possibles d'accroître la valeur de la variance expliquée, on parvient à l'élever, cette variance, bien sûr, mais seulement à $36 \%$. Huit variables sont sélectionnées, parmi lesquelles on ne trouve ni le sexe, ni le statut socioéconomique des parents. Toutes les corrélations sont faibles, presque toutes ramènent au bon sens. La première qui apparaît s'interprète aisément: plus on croit que les études universitaires sont trop théoriques, moins on se tourne vers les professions des niveaux supérieurs. La deuxième est d'une logique plus douteuse : plus est fréquent l'usage d'Internet pour la météorologie, plus on est 
Tableau 6

Régression multiple de diverses variables dont l'influence est apparue significative pour le niveau de la profession prisée.

Variance expliquée $\left(\mathrm{R}^{2}\right)$ et coefficient standardisé (ß)

\begin{tabular}{|c|c|c|c|}
\hline Variable déterminante sélectionnée & B & $\mathrm{F}$ & $\mathrm{p}<0,05$ \\
\hline Les études universitaires sont trop théoriques & $-0,43$ & 11,29 & Oui \\
\hline J'utilise Internet pour la météorologie & 0,28 & & \\
\hline $\begin{array}{l}\text { Je lis des journaux, des revues ou des magazines } \\
\text { dans une langue autre que le français ou l'anglais }\end{array}$ & $-0,19$ & & \\
\hline La plupart de mes ami-e-s pensent comme moi & $-0,18$ & & \\
\hline Dans tous les pays, la science se fait en anglais & 0,19 & & \\
\hline $\begin{array}{l}\text { Plus tard, je vivrai avec un-e conjoint-e, } \\
\text { mais je ne me marierai pas }\end{array}$ & 0,16 & & \\
\hline Je vais à la bibliothèque & 0,16 & & \\
\hline $\begin{array}{l}\text { J'ai beaucoup d'activités en dehors du cadre } \\
\text { de mes études et de mon travail }\end{array}$ & $-0,15$ & & \\
\hline \multicolumn{2}{|l|}{$\mathrm{R}^{2}$ total $=0,36$} & & \\
\hline
\end{tabular}

orienté vers les professions qui se situent au sommet; il faut retenir ici que l'usage d'Internet pour des fins météorologiques correspond en réalité à un usage généralisé du média. La troisième variable rappelle une certaine difficulté d'intégration des jeunes immigrants : plus l'élève lit dans une autre langue que celles qui sont officielles, moins il est interpellé par les postes prestigieux. La quatrième variable signale l'importance d'une certaine autonomie de l'esprit : plus le jeune estime que la plupart de ses amis pensent comme lui, moins il se destine aux plus hautes professions. « Dans tous les pays, la science se fait en anglais », plus on tend à le penser, plus l'attrait est grand pour les plus hautes fonctions. La sixième variable indique, elle aussi, timidement, l'importance d'une certaine autonomie de pensée : plus on tend à envisager la vie de couple en dehors du mariage, plus on est intéressé par les niveaux élevés. La septième variable montre que plus on va souvent à la bibliothèque, plus les aspirations professionnelles sont grandes. La huitième et dernière variable 
veut que plus une personne est active « en dehors du cadre de [s]es études et de [s]on travail », plus elle est ambitieuse. Il faut rappeler que toutes ces corrélations sont faibles, que toutes, donc, ne constituent que des probabilités ; il faut rappeler aussi qu'on n'a pas affaire à un type de personne, que, tout au plus, il s'agit de caractéristiques qui ne sont pas forcément reliées entre elles ; enfin, il faut souligner que toutes ces variables n'expliquent que $36 \%$ de la variance de l'aspiration professionnelle, que tout un univers de facteurs autres, donc, peut intervenir pour agir sur la manière dont le jeune conçoit son destin, ou même que les aspirations répondent à une tout autre logique.

\section{VI - INTERPRÉTATION ET CONCLUSION}

Longtemps, chez les jeunes, il fut plus fréquent, pour un FrancoOntarien que pour un Anglo-Ontarien, de se destiner aux études postsecondaires et surtout de se projeter dans les professions qui sont symboliquement les plus en vue. Ces aspirations étaient souvent utopiques en ce qu'elles ne coïncidaient pas avec une démarche susceptible de favoriser la réalisation des desseins ; elles ne suffisaient certainement pas à faire que les FrancoOntariens étudiassent davantage que les autres ou qu'ils occupassent plus souvent que les autres les plus hautes fonctions de la société ontarienne. Nous avions interprété cet idéalisme comme une manière de conjurer le sentiment d'aliénation qui était lié à leur situation de minoritaire. Mais les temps ont changé. La répartition des projets d'étude ou de carrière des FrancoOntariens ressemble maintenant à celle des Anglo-Ontariens ; l'idéologie culturelle ou ethnolinguistique des francophones s'apparente à celle des anglophones à cela près que les francophones valorisent un peu plus l'anglicité que les Anglais eux-mêmes, sont un peu plus pessimistes qu'eux quant à l'avenir de la francité, en Ontario et dans le monde. On peut supposer que cette transformation qui s'est effectuée au cours des quinze dernières années est à mettre en lien avec des tendances des sociétés industrielles avancées. Il faut sans doute évoquer 
l'homogénéisation qui est attribuable à la postindustrialisation, dont le corollaire est le rôle important que jouent les moyens de communication de masse. Les symboliques qui circulent dans ces sociétés transcendent souvent celles qui émanent des communautés spécifiques ${ }^{34}$. Certes, dans les années 1970 et 1980, il y avait des symboliques que partageaient les francophones et les anglophones de l'Ontario ; c'est pour cette raison, par exemple, que les francophones avaient pour ambition d'exercer des professions valorisées aussi bien par les uns que par les autres, des professions, donc, qui les rendraient admirables auprès des anglophones. Mais la postindustrialisation fait plus que cela : non seulement elle généralise les symboliques, mais elle uniformise aussi les conditions de la production de ces représentations. Elle le fait de plusieurs façons. Elle le fait en exposant les uns et les autres à des messages semblables. Elle le fait en favorisant un développement qui rend possible une ascension transgénérationnelle sur les plans éducationnel et professionnel. Mais cette évolution n'est pas automatique. Elle dépend, paradoxalement, de l'aptitude des collectivités particulières à se reproduire dans leur spécificité, nonobstant les tendances à l'indifférenciation. Or, cette reproduction dépend fortement du système institutionnel dont dispose une collectivité particulière. Raymond Breton a parlé pertinemment de « complé-tude institutionnelle». Dans la société postindustrielle, la reproduction des collectifs n'est possible que dans la mesure où ils bénéficient de l'ensemble des conditions grâce auxquelles ils peuvent exister en tant qu'ils participent de la postindustrialisation. Si, par exemple, ils ne rendent pas possible une formation universitaire au sein d'un système institutionnel propre alors que la postindustrialisation réclame des diplômés d'université, alors leur reproduction est menacée ; si la postmodernité suppose des messages de masse et que les collectifs ne peuvent en produire ou ne disposent pas de médias de masse,

34. Sur l'homogénéisation liée à la postindustrialisation et sur son corollaire, l'hétérogénéisation, voir Simon Laflamme et Ali Reguigui, Homogénéité et distinction, Sudbury, Prise de parole, coll. « Ancrages », 2003. 
alors ils sont à nouveau vulnérables. On peut donc concevoir que les Franco-Ontariens ont maintenant des aspirations comparables à celles des Anglo-Ontariens parce qu'ils s'inscrivent dans cette double tendance qui homogénéise les populations tout en rendant possible le renouvellement des particularismes. L'homogénéisation des populations sans que chacune d'elles dispose d'institutions qui lui soient propres est simple négation des particularismes ; elle est assimilation aux messages et aux conditions de vie de l'ensemble qui est le mieux pourvu sur le plan institutionnel. Cette assimilation est d'autant moins probable qu'une population s'inscrit dans le processus d'homogénéisation, qu'elle se dote d'un système institutionnel nécessaire aux sociétés post-industrielles, et donc qui soit commun à chacune d'elles, mais qui simultanément assure leur différenciation. Et ce sont sans doute les faiblesses de ce rapport aux institutions qui marginalisent les aspirations des jeunes des Premières Nations, lesquels peinent à se projeter vers l'université ou le font à certains égards s'ils admettent la nécessité de l'anglicité du monde. On ne sait pas si les aspirations des Franco-Ontariens se concrétiseront ; il faudra suivre les jeunes de notre étude pour le savoir ou vérifier plus tard si la structure occupationnelle des francophones en vient à ressembler à celle des anglophones ${ }^{35}$. On ne sait pas si les aspirations des francophones de l'Ontario procèdent de la même logique que celles des anglophones de la province; on sait cependant que les représentations ethnolinguistiques ne font pas varier sensiblement les projets de carrière. Le système institutionnel des francophones n'est certainement pas équivalent à celui des anglophones, celui-ci étant beaucoup plus développé et étendu que celui-là. Et cette inégalité représente certainement un danger pour la population francophone. Mais les formes et les niveaux de cette inégalité se sont très certainement modifiés au cours des dernières décennies et c'est à ce changement qu'on doit le fait

35. On sait que ce n'est pas le cas pour le moment, les Franco-Ontariens étant moins instruits que les Anglo-Ontariens, exerçant donc dans une plus grande proportion qu'eux les métiers du secteur primaire et travaillant dans de plus faibles proportions dans des postes de cadre. 
qu'il ne soit plus permis de dire que les ambitions des FrancoOntariens sont démesurées quand on les compare à celles des Anglo-Ontariens ; il faudra voir si ce changement aura eu pour effet que les desseins des Franco-Ontariens se matérialisent. Peutêtre qu'après avoir entretenu des rêves démesurés pendant des décennies, les Franco-Ontariens en viendront à se donner une société sur mesure!

Le rôle que joue l'ambition dans l'imaginaire franco-ontarien et les modifications de ce rôle sont à mettre en relation avec l'évolution de la société postindustrielle. Cela a pour conséquence, outre que, en Ontario, les projets des francophones ressemblent à ceux des anglophones, que ni les perspectives du jeune, ni ce qu'il devient réellement soient prédéterminés par la scolarité ou par le travail des parents. Cela a pour effet également que les niveaux prisés varient peu selon le sexe. Mais cela ne veut pas dire que garçons et filles rêvent nécessairement des mêmes choses ; cela signifie qu'ils peuvent le faire. Cela ne suppose pas que les parents n'ont pas d'influence sur leurs enfants; cela implique que cette influence ne constitue pas un programme, qu'elle est relativisée par l'ensemble des sources auprès desquelles un jeune peut s'informer et par la complexification de la circulation de l'information dans les sociétés postindustrielles. L'ethnie peut jouer un rôle, mais ce rôle est d'autant plus marquant qu'il correspond à une discrimination, c'est-à-dire qu'il se présente sous la forme d'une inégalité des systèmes institutionnels. Tout cela rappelle que l'avenir de chacun des jeunes est ouvert, mais qu'il est nécessaire que la société à laquelle chacun d'eux appartient se donne les moyens des ambitions de ses jeunes. Notre analyse de régression a éliminé l'instruction et l'occupation des parents, de même que le sexe comme déterminants des aspirations professionnelles. Ces variables ont été supplantées par un ensemble qui rappelle la force du rapport à l'information; sont apparus dans l'équation, en effet, l'usage d'Internet et la fréquentation d'une bibliothèque. Si faibles que soient ces corrélations, elles signalent l'importance d'exposer les jeunes aux 
nouveaux médias et de favoriser le rapport aux lieux dans lesquels sont déposés les écrits. Les variables qui sont liées à l'origine familiale ont aussi dû faire place à des facteurs comme l'idéologie : l'anti-intellectualisme réduit la propension à s'instruire; la société qui l'encourage empêche ses jeunes de participer de la postindustrialisation. Ces variables classiques ont été écartées par d'autres qui montrent l'importance d'avoir des amis, mais aussi d'éprouver une certaine latitude de la pensée. Elles ont cédé le pas à des considérations qui mettent en évidence la nécessité de l'intégration sociale en même temps que l'autonomie, par des éléments qui soulignent la nécessité pour un jeune d'avoir beaucoup d'activités dans sa communauté, de vivre pleinement, même en dehors du cadre de ses études et de son travail. Ces constats ne peuvent que rappeler à l'ordre toute personne qui entend travailler au développement communautaire et favoriser le développement intellectuel des populations. Mais on se souviendra que, en intégrant les jeunes tout en admettant leur autonomie, en offrant des médias et des institutions du savoir, en faisant en sorte que les jeunes apprécient leur société et s'y investissent, on offre des conditions favorables au devenir, mais qu'il ne s'agit pas là de conditions suffisantes. 